\title{
Dilepton Enhancement by Thermal Pion Annihilation in the CERES Experiment
}

\author{
H.-J. Schulze and D. Blaschke \\ MPG-AG "Theoretische Vielteilchenphysik", Universität Rostock, D-18051 Rostock, Germany
}

\begin{abstract}
We compare the recent CERES data on dielectron production in 200 $\mathrm{GeV} / \mathrm{u} \mathrm{S}+\mathrm{Au}$ collisions with the theoretical predictions due to pion annihilation in a thermal hadronization and a string fragmentation scenario. Both models yield similar results for the dilepton mass spectrum. A satisfactory description of the experimental spectrum requires an in-medium reduction of the rho-mass and a freeze-out temperature of about $150 \mathrm{MeV}$ in the thermal model. We emphasize and discuss the influence of experimental acceptance and resolution corrections.
\end{abstract}

The recent results of the CERES collaboration on the production of electron pairs in $\mathrm{S}+\mathrm{Au}$ collisions at $200 \mathrm{GeV} / \mathrm{u}$ [1,2] allow for the first time a quantitative comparison with theoretical models for this type of ultrarelativistic heavy ion collisions. The CERES collaboration reports a five-fold enhancement of the dilepton spectrum in the mass range from the two-pion threshold up to $1.5 \mathrm{GeV}$, compared to the expectation based on an independent superposition of nucleon-nucleon collisions. Their interpretation of this enhancement is being due to the production of dileptons from pion-pion annihilation in the hot and dense medium that is supposedly formed during the collision. The shape of the observed spectrum then hints to a modification of the pion annihilation form factor in this medium. There have been a couple of quantitative analyses of the results based on relativistic transport models [3 5]. These works are able to describe the data reasonably well using a suitable medium-modified form factor.

On the other hand, thermal models have been used successfully to describe observables like hadron abundances [6] and hadron spectra [7] in ultrarelativistic heavy ion collisions. While giving relatively good fits to the data, they have the advantage of simplicity and and a small number of adjustable parameters, the most prominent one being a "freeze-out" temperature $T_{f}$.

In this paper we would like to analyze the dilepton production within a similarly simple thermal hadronization model, the standard "benchmark" scenario based on the Bjorken model of a boost-invariant longitudinal expansion [8], including a quark phase $(u+d+g)$, a mixed phase, and a hadronic pion gas phase. It is characterized by three temperatures, the formation temperature $T_{i}$ of the quark phase, the critical temperature $T_{c}$ for the hadronization in a mixed phase of quarks and pions, and the freeze-out temperature $T_{f}$. This scenario 
has often been employed to predict the dilepton production rate in ultrarelativistic heavy ion collisions [9, 10], until recently however without a chance of quantitative experimental comparison.

A first test of the model will be the comparison with a purely hadronic scenario based on the pion distribution functions as obtained from a string-fragmentation event generator (in this case VENUS) [11. Both models should yield similar results for the (unmodified) dilepton mass spectrum, because at present collision energies the quark annihilation contribution in the thermal model is negligible compared to the pion annihilation part [9]. This supposition is indeed confirmed by our results. The models therefore provide a reliable basis upon which more sophisticated features like medium-dependent properties of pion [12] or rho-meson [13 15], finite pion [16 18] or quark [19] chemical potentials, transverse expansion [19,20], or more exotic possibilities [21], can be studied. We will then use the thermal model in order to test the influence of a medium modification of the pion form factor. With the simple model of an unmodified form factor in the hadronic phase and a dropping rho-mass in the mixed phase we are able to achieve a very satisfactory fit of the dilepton spectrum when the thermal history of the hot matter is described by a set of temperatures $\left(T_{i}, T_{c}, T_{f}\right)=(250,160,150) \mathrm{MeV}$.

Before a detailed description of the models, we start however with a discussion of the general "kinematical" conditions of the experiment. Obviously, in any comparison with a theoretical model, the same kinematical cuts and detector resolutions as in the actual experiment have to be applied. In this case, they are fairly severe: A dilepton is only detected, if both the electron and the positron momenta satisfy the kinematical restrictions on pseudorapidity, transverse momentum, and opening angle,

$$
\begin{aligned}
& 2.1<\eta_{e}<2.65, \\
& p_{T}^{(e)}>0.2 \mathrm{GeV}, \\
& \Theta_{e e}>35 \mathrm{mrad}=2^{\circ} .
\end{aligned}
$$

These conditions translate into an acceptance function $A\left(M, q_{T}\right)$ depending on the mass $M$ and transverse momentum $q_{T}$ of the virtual photon that decays into the lepton pair. A first guess can be made by neglecting the transverse momentum. The acceptance for an isotropic decay of the virtual photon in its rest frame (as appropriate for a thermal production model) is then simply given by

$$
A(M, 0) \approx \theta(M-0.4 \mathrm{GeV}) \frac{\log [\cosh (0.275)]}{0.275} .
$$

The numerical value of the second factor is 0.136 , and the observed spectrum is therefore reduced by nearly an order of magnitude. The full acceptance function can be computed numerically and is shown in Fig. [. (In fact it turns out that for two-body decays the restriction on the opening angle Eq. (11) has only an effect for extremely low masses $M<$ $50 \mathrm{MeV}$, as displayed in the figure). It can be seen that there is a strong variation with transverse momentum, in particular for low masses that we are interested in. The notion of an acceptance function $A(M)$ depending only on the dilepton mass $M$, is therefore a strong approximation. For a proper treatment the theoretical spectrum $d N_{e e} / d^{4} q$ has to be multiplied with the acceptance function $A\left(M, q_{T}\right)$, before the mass spectrum $d N_{e e} / d M d y$ 
can be computed by integration over $q_{T}$. Before a comparison with the experimental data, the theoretical spectrum has still to be folded with a Gaussian representing the finite mass resolution $\delta M / M$ of the detector, which is approximately given by the formula [2]:

$$
\frac{\delta M}{M}= \begin{cases}0.08 & , \quad M<0.5 \mathrm{GeV} \\ 0.03+0.1 M / \mathrm{GeV} \quad, \quad M>0.5 \mathrm{GeV} .\end{cases}
$$

Finally, for a careful comparison it is important to note that the CERES result represents an average over an ensemble of events with mean charged particle multiplicity $\left\langle d N_{\mathrm{ch}} / d y\right\rangle \approx 125$ and RMS value $\sqrt{\left\langle\left(d N_{\mathrm{ch}} / d y\right)^{2}\right\rangle} \approx 135$ [2].

We come now to the detailed description of both scenarios of pion annihilation in order to compute the dilepton spectrum $d N_{e e} / d^{4} q$. Starting with a short review of the thermal model, the production rate of electron pairs with four-momentum $q$ from the relativistic quark or pion gas at temperature $T$ is

$$
\frac{d N_{e e}}{d^{4} x d^{4} q}=\frac{M^{2} a \sigma(M)}{4 \pi(2 \pi)^{4}} F(M, E, T)
$$

with $M^{2}=q^{2}, a=\sqrt{1-4 m^{2} / M^{2}},\left(m=m_{q}, m_{\pi}\right)$, and the elementary cross sections in the quark $(\mathrm{Q})$ and hadronic $(\mathrm{H})$ phases,

$$
\begin{aligned}
a \sigma(M)=\frac{4 \pi \alpha^{2}}{3 M^{2}} \widetilde{\sigma}(M) \quad, \quad \widetilde{\sigma}_{Q}(M) & =\frac{20}{3}\left(1+\frac{2 m_{q}^{2}}{M^{2}}\right)\left(1+\frac{2 m_{e}^{2}}{M^{2}}\right) \sqrt{1-\frac{4 m_{e}^{2}}{M^{2}}}, \\
\widetilde{\sigma}_{H}(M) & =\left|F_{\pi}(M)\right|^{2}\left(1-\frac{4 m_{\pi}^{2}}{M^{2}}\right)\left(1+\frac{2 m_{e}^{2}}{M^{2}}\right) \sqrt{1-\frac{4 m_{e}^{2}}{M^{2}}}
\end{aligned}
$$

with the pion form factor

$$
\left|F_{\pi}(M)\right|^{2}=\frac{C m_{\rho}^{4}+m_{\rho}^{2} \Gamma_{\rho}^{2}}{\left(M^{2}-m_{\rho}^{2}\right)^{2}+m_{\rho}^{2} \Gamma_{\rho}^{2}}
$$

and $C=1.3, m_{\rho}=760 \mathrm{MeV}, \Gamma_{\rho}=135 \mathrm{MeV}$. (These parameters fit the experimental data 22] better than the standard choice $C=1, m_{\rho}=770 \mathrm{MeV}, \Gamma_{\rho}=160 \mathrm{MeV}$ ).

The function $F(M, E, T)$ in Eq. (4) is given by a phase-space integral over the Fermi (Bose) distributions of the reacting quarks (pions) 10,17:

$$
\begin{aligned}
& F_{Q}(M, E, T)=\frac{1}{\beta \bar{q}} \frac{1}{\exp (\beta E)+1} \log \left[\frac{\cosh \beta \omega_{\max }+\cosh \beta \mu_{q}}{\cosh \beta \omega_{\min }+\cosh \beta \mu_{q}}\right], \\
& F_{H}(M, E, T)=\frac{1}{\beta \bar{q}} \frac{1}{\exp \left(\beta E-2 \beta \mu_{\pi}\right)-1} \log \left[\frac{\cosh \beta\left(\omega_{\max }-\mu_{\pi}\right)-1}{\cosh \beta\left(\omega_{\min }-\mu_{\pi}\right)-1}\right] .
\end{aligned}
$$

Here $E=q \cdot u(x)$ is the dilepton energy, and $\bar{q}=\sqrt{E^{2}-M^{2}}$ is the three-momentum component of $q$ in the production fluid element moving with four-velocity $u$. $\omega_{\max , \min }=$ $(E \pm a \bar{q}) / 2$ is the maximal (minimal) quark or pion energy in this frame. The formula includes finite chemical potentials for the quarks [19] and the pions [16, 17], although we restrict ourselves in the following to the case $\mu_{q}=\mu_{\pi}=0$. The integration of the rate over space-time then gives the momentum spectrum of lepton pairs: 


$$
\begin{aligned}
& \frac{d N_{e e}}{d^{4} q}=\pi R^{2} \int_{0}^{\infty} d \tau \tau \int_{-\infty}^{\infty} d \eta \frac{d N_{e e}}{d^{4} x d^{4} q} \\
& =\frac{\alpha^{2}}{16 \pi^{5}} \frac{\left(\pi R^{2} \tau_{c} T_{c}^{3}\right)^{2}}{R^{2} M_{T}^{6}} \times \\
& \left\{\widetilde{\sigma}_{Q}(M)\left[\int_{M_{T} / T_{i}}^{M_{T} / T_{c}} d z z^{5} G_{Q}\left(M, M_{T}, T=M_{T} / z\right)+\frac{r-1}{6} z_{c}^{6} G_{Q}\left(M, M_{T}, T_{c}\right)\right]+\right. \\
& \left.\tilde{\sigma}_{H}(M)\left[r^{2} \int_{M_{T} / T_{c}}^{M_{T} / T_{f}} d z z^{5} G_{H}\left(M, M_{T}, T=M_{T} / z\right)+r \frac{r-1}{6} z_{c}^{6} G_{H}\left(M, M_{T}, T_{c}\right)\right]\right\},
\end{aligned}
$$

where $r=37 / 3$ is the ratio of degrees of freedom in the quark and hadronic phases, and $G$ represents the integral over space-time rapidity:

$$
G\left(M, M_{T}, T\right)=\left.\int_{-\infty}^{\infty} d \eta F(M, E, T)\right|_{E(\eta)=M_{T} \cosh (\eta-y)}
$$

It sums the dileptons produced in different slices of space-time rapidity $\eta$ at given propertime $\tau$ (and temperature $T$ ). The energy $E$ of the dilepton in these local production frames is related by a Lorentz-boost to the energy in the lab system, $M_{T} \cosh y$. The normalization of the spectrum can be linked to the square of the pion rapidity density by employing the relation $\pi R^{2} \tau T^{3}=\kappa d N_{\pi} / d y$, with $\kappa \approx 0.22$ in the quark phase. The final result is

$$
\frac{d N_{e e}}{d^{4} q}=\frac{\alpha^{2}}{16 \pi^{5}} \frac{\left(\kappa d N_{\pi} / d y\right)^{2}}{R^{2}} \frac{1}{M_{T}^{6}}\{\ldots\} \approx \frac{7 \times 10^{-8} \mathrm{GeV}^{2}}{M_{T}^{6}}\{\ldots\},
$$

where the expression in curly brackets is the one given in Eq. (9). In the last step we have inserted the appropriate numerical values $R \approx R_{S} \approx 18 \mathrm{GeV}^{-1}$, and $\left\langle d N_{\pi} / d y\right\rangle_{\mathrm{RMS}} \approx 200$ [2].

The spectrum is now completely determined by specifying the three temperatures $T_{i}, T_{c}$, and $T_{f}$, and we use here for the moment a standard set of $T_{i}=250 \mathrm{MeV}, T_{c}=160 \mathrm{MeV}$, and $T_{f}=120 \mathrm{MeV}$, that we will discuss later. The comparison with the experimental mass spectrum can then be done by multiplying the theoretical spectrum with the acceptance function, integrating over the transverse momentum, and folding with the mass resolution of the detector, as discussed above. The result is shown in Fig. 2, where we display the spectrum before any correction, after acceptance correction, and the final result. The comparison of the three curves demonstrates the importance of the corrections. In particular the slope of the mass spectrum beyond the rho-peak seems to be entirely determined by the finite mass resolution. Before a further discussion of the figure, we turn however to the second, completely hadronic scenario of dilepton production, namely by pion annihilation in a string fragmentation model.

We start by repeating the expression for the dilepton spectrum found in Ref. [11]:

$$
\frac{d N_{e e}}{d^{4} q}=\frac{16 \alpha^{2}}{27\left\langle p_{T}\right\rangle^{4}\left\langle m_{T}\right\rangle}\left[\int_{0}^{\infty} d \tau \frac{d N_{\pi}}{d y}(y, \tau) \epsilon_{\pi}(y, \tau)\right] \frac{1}{\cosh ^{4}(y)} \frac{\tilde{\sigma}_{H}(M) \exp \left(-M_{T} / a\right)}{M M_{T}}
$$

with $d^{4} q=M d M d y d^{2} q_{T}$. This result was obtained by assuming that throughout the reaction the pions are produced with a momentum distribution $d N_{\pi} / d y d p_{T}^{2} \sim \delta(y-\eta) \exp \left(-m_{T} / a\right)$. The parameters $\left\langle p_{T}\right\rangle$ and $\left\langle m_{T}\right\rangle$ are the average transverse momentum and transverse mass 
of the pions. For further information on the assumptions of the model, and details of the calculation, we refer to Ref. [11].

The dynamical content of Eq. (12) is the one-dimensional integral of the temporal development of the product of particle density $d N_{\pi} / d y$ and energy density $\epsilon_{\pi}=$ $\left\langle m_{T}\right\rangle /\left(\tau \pi R^{2}\right) d N_{\pi} / d y$ in a given slice of rapidity,

$$
I=\int_{0}^{\infty} d \tau \frac{d N_{\pi}}{d y}(y, \tau) \epsilon_{\pi}(y, \tau) .
$$

This integral contains the dependence on energy and mass number of the colliding nuclei. In Ref. [1] we used the results of the VENUS event generator [23] on particle and energy densities for central $\mathrm{O}+\mathrm{Au}$ collisions at $200 \mathrm{GeV} / \mathrm{u}$ [24] and found an upper limit of $40 \mathrm{GeV}^{3}$ for this integral. Unfortunately, new results of VENUS on these observables for the case of a $\mathrm{S}+\mathrm{Au}$ reaction are not available. We therefore employ here a simple scaling assumption and multiply the $\mathrm{O}+\mathrm{Au}$ result with a factor

$$
\left(\frac{\left(d N_{\pi} / d y\right)_{S}}{\left(d N_{\pi} / d y\right)_{O}}\right)^{2} \times \frac{R_{O}^{2}}{R_{S}^{2}} \approx 1.1,
$$

with $\left(d N_{\pi} / d y\right)_{O} \approx 150$ and $\left(d N_{\pi} / d y\right)_{S} \approx 200$ appropriate for the two cases. Considering the accuracy of the whole model, this procedure should certainly be adequate. Further we use in Eq. (12) a kinematical factor $\cosh ^{4}(y) \approx 2.1$, since the center of the CERES rapidity coverage lies about 0.6 units away from the nucleon-nucleon cms rapidity (3.0). The resulting dilepton spectrum, with an average transverse momentum of pions $\left\langle p_{T}\right\rangle \approx 0.4 \mathrm{GeV}$ [25], and after acceptance and resolution corrections, is also shown in Fig. 2 .

It is immediately noted that although the two scenarios considered here, thermal and string fragmentation, rest on completely different physical assumptions and operate on different time scales, their predictions, at least for the mass spectrum of dileptons, are nearly undistinguishable (apart from the quark phase contribution in the thermal model at very low masses, which is however completely negligible in the present type of reaction). This shows that on the one hand the dilepton mass spectrum is unfortunately rather insensitive to the underlying hadron (pion) dynamics, on the other hand it allows us to use now the thermal model with some confidence in order to adjust parameters and test assumptions about medium modifications.

Concerning the influence of the choice of temperatures $T_{i}, T_{c}, T_{f}$, we find that a variation of $T_{i}$ has practically no importance, since the quark part of the spectrum is negligible anyway. The value of the critical temperature $T_{c}=160 \mathrm{MeV}$ appears generally accepted, and we fix it as a "canonical" value. A variation of $T_{f}$ within a relevant range $100 \ldots 150 \mathrm{MeV}$ changes the spectrum by about a factor two, but preserves its overall shape. A low value of $T_{f}=120 \mathrm{MeV}$, as used in Fig. 2, is a more "traditional" choice [9, 10], whereas rather large values are supported by recent fits to hadron abundances in the present experiment $\left(T_{f} \approx 160-170 \mathrm{MeV}\right.$, [6] ) or to slopes of hadron spectra $\left(T_{f} \approx 150 \mathrm{MeV},[7]\right)$.

We find then that with $T_{f}=120(150) \mathrm{MeV}$ and using the free pion form factor the theoretical predictions exceed the CERES data by about a factor four (three) in the rhomass region. In comparison, in Refs. 团 and [5] that are based on more sophisticated transport models, the corresponding enhancement factors are approximately three and two, respectively. We can now conclude that a sizable finite chemical potential of the pions 
[16.17] can probably be excluded by our result, since this would again enhance the dilepton spectrum by nearly an order of magnitude, as shown in Ref. [17].

A possibility of reducing the theoretical expectation is of course the in-medium modification of the elementary cross sections and form factors, in particular for the rho meson [26].27]. The pion is thought to be rather stable against medium effects [12], and we do not consider a modification here. Unfortunately, the numerous models on the modification of the rho meson [13 [15] do not even agree on the simplest qualitative aspects of the variation of, say, the rho-mass $m_{\rho}$ and width $\Gamma_{\rho}$ in the medium.

We do not intend to introduce a further model here. Instead we will use two simple parametrizations of the temperature dependences of $m_{\rho}$ and $\Gamma_{\rho}$ in the form factor Eq. (6) together with the thermal scenario described above in order to assess the principle effects on the dilepton spectrum.

The first parametrization is the one employed in Ref. [26], namely an increase of the width with temperature, while the mass is kept constant: $\Gamma_{\rho}(T)=\Gamma_{\rho} /\left(1-T^{2} / T_{c}^{2}\right)$. This means that in the mixed phase the pion-pion cross section is reduced to the Born term, while in the hadronic phase it is still substantially suppressed. This is probably unrealistic, and the results should therefore be considered as a lower bound on what a medium modification of the form factor can achieve.

The second parametrization is based on the assumption that the vector meson mass does not change dramatically in a hot hadronic medium and is during the hadronic phase given by its vacuum value [14]. However, in the mixed phase at the critical temperature for the deconfinement transition $T_{c}=160 \mathrm{MeV}$, the mass might be lowered as conjectured by a number of authors [15]. Here we simply assume a mass of $m_{\rho}\left(T_{c}\right)=m_{\rho} / 2 \approx 380 \mathrm{MeV}$. Although at present there is no firm theoretical foundation for such an assumption, we consider it as a guess which allows to reproduce the two-peak structure of the experimental spectrum.

We display the results in Figs. [3, 回. For a better comparison with the data, we have added here the part of the dilepton spectrum due to hadron decays, as given in Ref. [1], to our pion annihilation results. Indeed the spectrum below $200 \mathrm{MeV}$ is well explained by the Dalitz decays of $\pi_{0}, \eta$, and $\omega$ mesons. We observe then that the varying-width scenario that we consider as a lower bound, is in good agreement with the overall normalization of the data using $T_{f}=120 \mathrm{MeV}$, whereas the dropping-mass model at $T_{f}=150 \mathrm{MeV}$ gives a rather good fit to the shape of the spectrum, in fact of the same quality as obtained with sophisticated transport models and more realistic medium modifications [3 [5]. This shows that the larger freeze-out temperature of $150 \mathrm{MeV}$ is better suited to fit the data, in agreement with the fairly large freeze-out temperatures deduced from the fit of hadron abundances [6] in the same experiment. A suitable in-medium modification of the pion form factor is thus indeed able to account for the observed spectrum.

This statement is valid with the exception of the data point at $M=0.25 \mathrm{MeV}$, i.e., below the two-pion threshold, that neither in our nor in previous models [3 5] can be reproduced in a satisfactory manner. This hints to the possibility of a non-negligible modification of the pion dispersion relation in the medium, but also the Dalitz decay of the $\eta$ meson is still important in this region and should be reconsidered.

We conclude that pion annihilation can indeed account for the observed excess of dileptons, as already advocated by the CERES collaboration [ [ 1 ]. We have presented a detailed 
adaptation of both a simple Bjorken scenario for the thermal dilepton production, as well as of a purely hadronic scenario due to string fragmentation, to the experimental situation. These models have the advantage of transparency and simplicity, but can not be expected to give perfect fits to the data. Nevertheless, we find that both models give surprisingly similar results (which means that the possible existence of a quark-gluon plasma can neither be affirmed nor rejected from the present data), and can account for the experimental spectrum by assuming a dropping rho-mass in the medium. The justification of such an assumption remains a theoretical challenge. We have here merely provided a guess that allows to reproduce the data. A freeze-out temperature of about $150 \mathrm{MeV}$ is supported by the experimental results. The correct implementation of acceptance and resolution corrections has been seen to be extremely important.

In particular with a large freeze-out temperature of about $150 \mathrm{MeV}$ nearly all dileptons then stem from pion annihilation in the rather long-lived mixed phase (of duration $(r-1) \tau_{c} \approx$ $24 \mathrm{fm} / c$ in the one-dimensional expansion scenario, and with the parameters chosen here). This puts forward the question of physical reality of such a phase. First principle, i.e., lattice gauge calculations do not yet provide an unanimous answer to this problem [28]. It is possible that a first order transition does not occur at all, or that the release of latent heat is fairly small. In any case there exists a relatively narrow transition region between the phases, in which hadronic correlations persist. The simple model of a first order phase transition between ideal quark and hadron gases is certainly an oversimplification, and should be considered a phenomenological approach to mimic the features of this real situation. The same remark applies of course to other features of the computation, such as the treatment of collision dynamics or medium modification of hadron parameters, as discussed before.

We would finally like to add a speculative estimate of the expected dilepton spectrum in central $\mathrm{Pb}+\mathrm{Au}$ (or $\mathrm{Pb}+\mathrm{Pb}$ ) collisions [29]. In both models presented here, the dominant source of change is a scaling factor $\left(d N_{\pi} / d y\right)^{2} / R^{2}$, cf. Eq. (14). For example, with $\left(d N_{\pi} / d y\right)_{\mathrm{Pb}} \approx 700$ we would obtain a rescaling of the curves presented in this paper by about a factor $12 / 3.4 \approx 3.5$. Of course, this scaling factor applies only with identical acceptance cuts as in the present experiment, so that in practice a completely new analysis suited to the experimental conditions will have to be done.

We would like to thank A. Drees and Th. Ullrich for discussions and detailed information on the setup of the CERES experiment. We thank G. Röpke for a critical reading of the manuscript. D.B. acknowledges enlightening discussions with K. Redlich. 


\section{REFERENCES}

[1] CERES Collab., G. Agakichiev et al., Phys. Rev. Lett. 75, 1272 (1995).

[2] CERES Collab., A. Drees and Th. Ullrich, Internal Memorandum, (1995).

[3] W. Cassing, W. Ehehalt, and C. M. Ko, Phys. Lett. B 363, 35 (1995).

[4] G. Q. Li, C. M. Ko, and G. E. Brown, Phys. Rev. Lett. 75, 4007 (1995).

[5] G. Chanfray, R. Rapp, and J. Wambach, Phys. Rev. Lett. 76, 368 (1996).

[6] P. Braun-Munzinger, J. Stachel, J. P. Wessels, and N. Xu, Phys. Lett. B 365, 1 (1996).

[7] U. Heinz, in: Hot Hadronic Matter: Theory and Experiment, Edited by J. Letessier et al., Plenum Press, New York, 1995.

[8] J. D. Bjorken, Phys. Rev. D 27, 140 (1983).

[9] E. L. Feinberg, Nuov. Cim. 34 A, 391 (1976);

G. Domokos and J. I. Goldman, Phys. Rev. D 23, 203 (1981);

G. Domokos, Phys. Rev. D 28, 123 (1983);

L. D. McLerran and T. Toimela, Phys. Rev. D 31, 545 (1985);

R. C. Hwa and K. Kajantie, Phys. Rev. D 32, 1109 (1985);

K. Kajantie, J. Kapusta, L. McLerran, and A. Mekjian, Phys. Rev. D 34, 2746 (1986);

K. Kajantie and P. V. Ruuskanen, Z. Phys. C 44, 167 (1989).

[10] J. Cleymans, J. Fingberg, and K. Redlich, Phys. Rev. D 35, 2153 (1987);

B. Kämpfer, O. P. Pavlenko, M. I. Gorenstein, A. Peshier, G. Soff, Z. Phys. A 353, 71 (1995).

[11] H.-J. Schulze, Phys. Lett. B 244, 507 (1990).

[12] C. L. Korpa and S. Pratt, Phys. Rev. Lett. 64, 1502 (1990);

A. Schenk, Nucl. Phys. B 363, 97 (1991);

G. E. Brown, V. Koch, and M. Rho, Nucl. Phys. A 535, 701 (1991);

R. Rapp and J. Wambach, Phys. Lett. B 315, 220 (1993);

D. Blaschke et al., Nucl. Phys. A 586, 711 (1995).

[13] G. Chanfray, Z. Aouissat, P. Schuck, and W. Nörenberg, Phys. Lett. B 256, 325 (1991);

G. Chanfray and P. Schuck, Nucl. Phys. A 545, 271c (1992).

[14] M. Herrmann, B. L. Friman, and W. Nörenberg, Nucl. Phys. A 560, 411 (1993);

C. Song, Phys. Rev. D 53, 3962 (1996).

[15] G. E. Brown and M. Rho, Phys. Rev. Lett. 66, 2720 (1991);

T. Hatsuda and S. H. Lee, Phys. Rev. C 46, R34 (1992).

[16] M. Kataja and P. V. Ruuskanen, Phys. Lett. B 243, 181 (1990).

[17] B. Kämpfer, P. Koch, and O. P. Pavlenko, Phys. Rev. C 49, 1132 (1994).

[18] A. I. Titov, T. I. Gulamov, and B. Kämpfer, Phys. Rev. D 53, 3770 (1996).

[19] B. Kämpfer, O. P. Pavlenko, A. Peshier, G. Soff, Phys. Rev. C 52, 2704 (1995).

[20] K. Kajantie, M. Kataja, L. McLerran, and P. V. Ruuskanen, Phys. Rev. D 34, 811 (1986).

[21] H. A. Weldon, Phys. Rev. D 42, 2384 (1990);

H. A. Weldon, Phys. Lett. B 274, 133 (1992).

[22] A. Quenzer et al., Phys. Lett. B 76, 513 (1978);

S. R. Amendolia et al., Phys. Lett. B 138, 454 (1984);

L. M. Barkow et al., Nucl. Phys. B 256, 365 (1985);

DM2 Collab., D. Bisello et al., Phys. Lett. B 220, 321 (1989).

[23] K. Werner, Phys. Rep. 232, 87 (1993). 
[24] K. Werner, Phys. Lett. B 219, 111 (1989).

[25] WA80 Collab., R. Santo et al., Nucl. Phys. A 566, 61c (1994);

A. Drees et al., Nucl. Phys. A 566, 87c (1994).

[26] C. A. Dominguez and M. Loewe, Z. Phys. C 49, 423 (1991).

[27] F. Karsch, K. Redlich, L.Turko, Z. Phys. C 60, 519 (1993);

Chungsik Song, Su Houng Lee, and Che Ming Ko, Phys. Rev. D 52, R476 (1995).

[28] F. Karsch and E. Laermann, Rep. Prog. Phys. 56, 1347 (1993).

T. Blum, L. Kärkkäinen, D. Toussaint, and S. Gottlieb, Phys. Rev. D 51, 5153 (1995).

[29] NA49 Collab., S. Margetis et al., in: Quark Matter 95, Nucl. Phys. A 590, 355c (1995). 


\section{FIGURES}

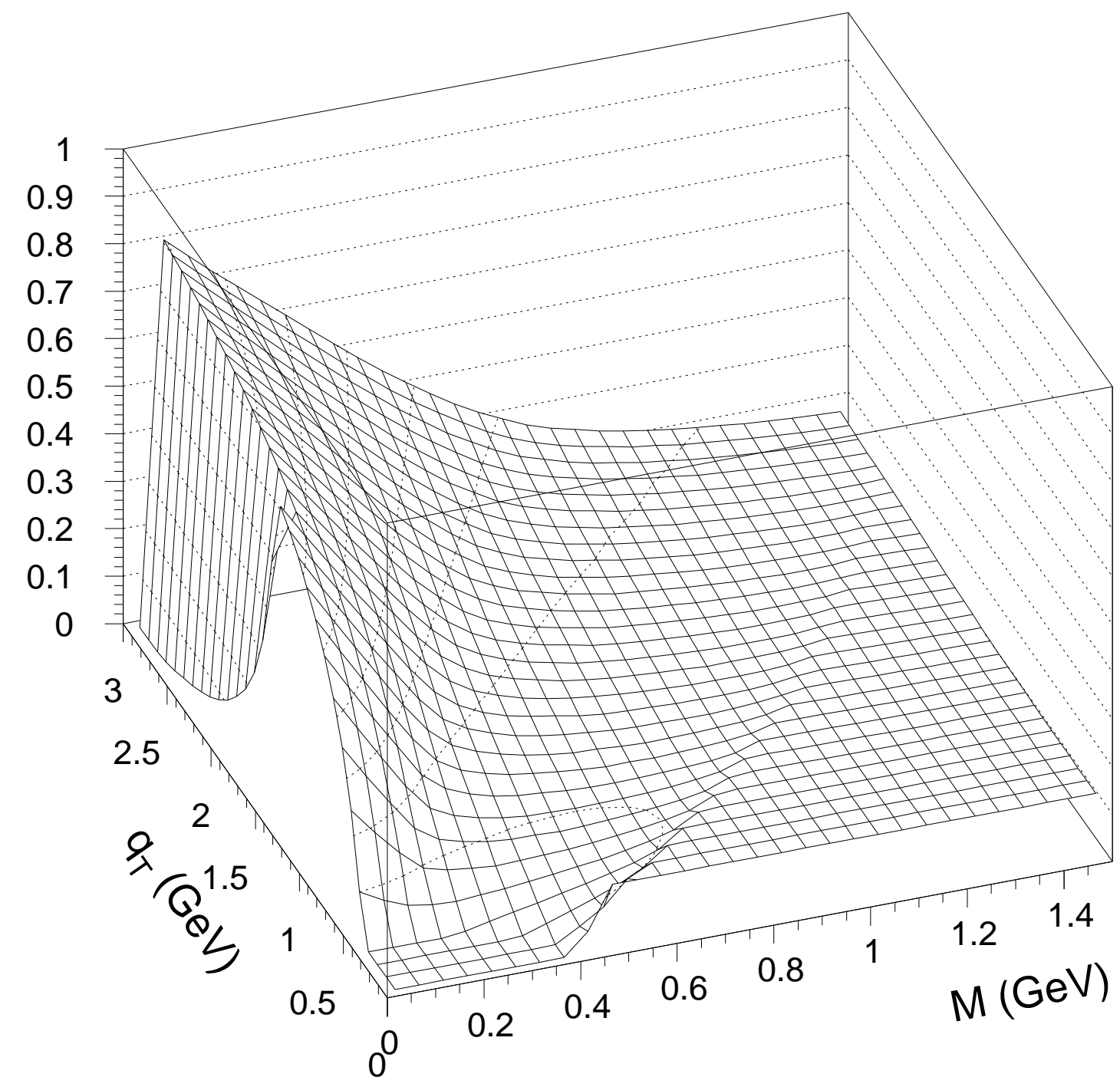

FIG. 1. The acceptance function for a virtual photon with mass $M$ and transverse momentum $q_{T}$ decaying into an electron pair subject to the kinematical restrictions Eq. (1). 


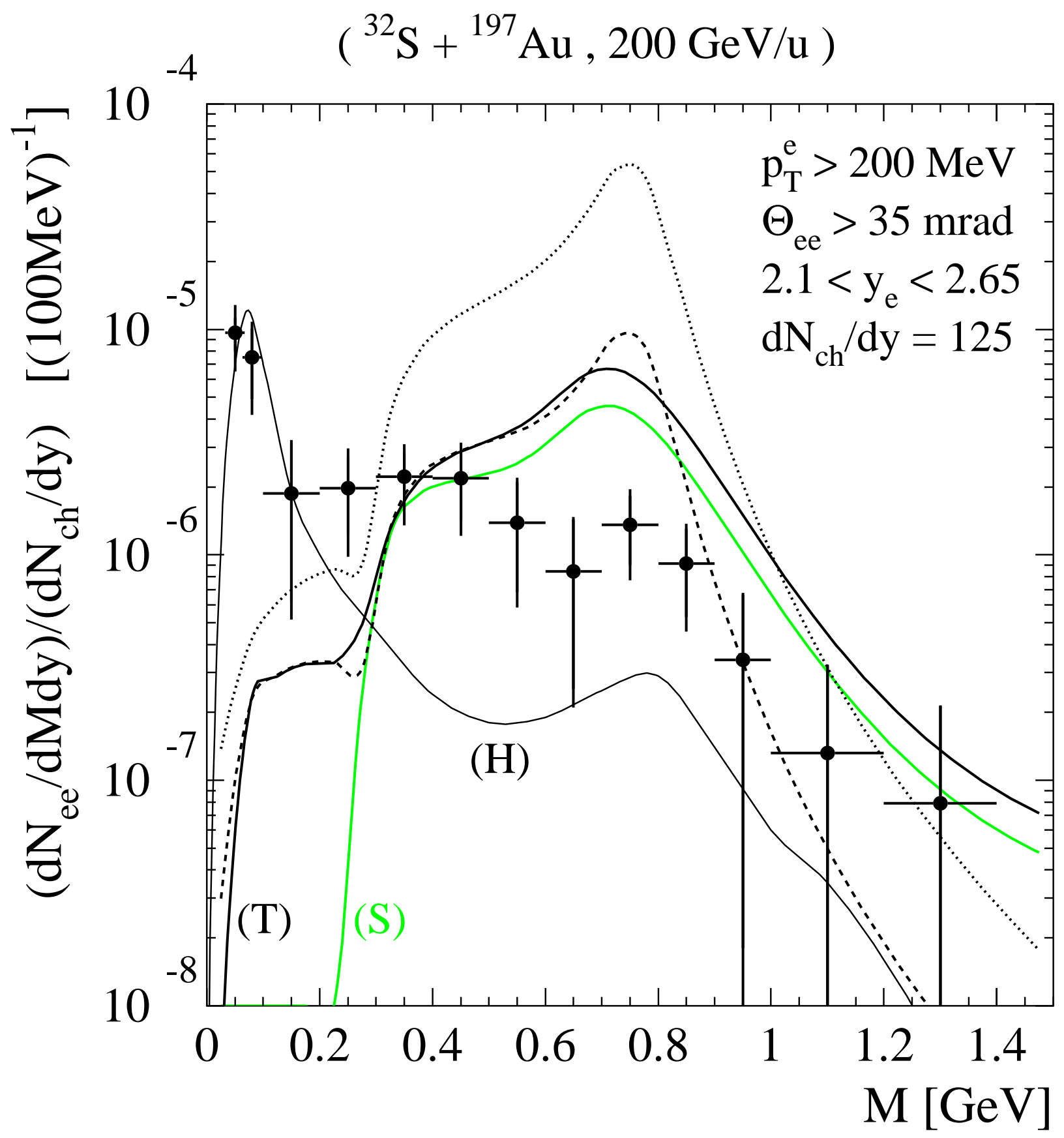

FIG. 2. The dilepton mass spectrum. The full lines represent the theoretical predictions in the thermal $(\mathrm{T})$ and the string model $(\mathrm{S})$ scenario after acceptance and resolution corrections. For the thermal scenario, we plot also the results before resolution correction (dashed line) and before acceptance correction (dotted line). The data are taken from Ref. [1]. (Statistical and systematical errors added quadratically). The curve denoted $(\mathrm{H})$ shows the total hadron decay contribution as given in that reference. 


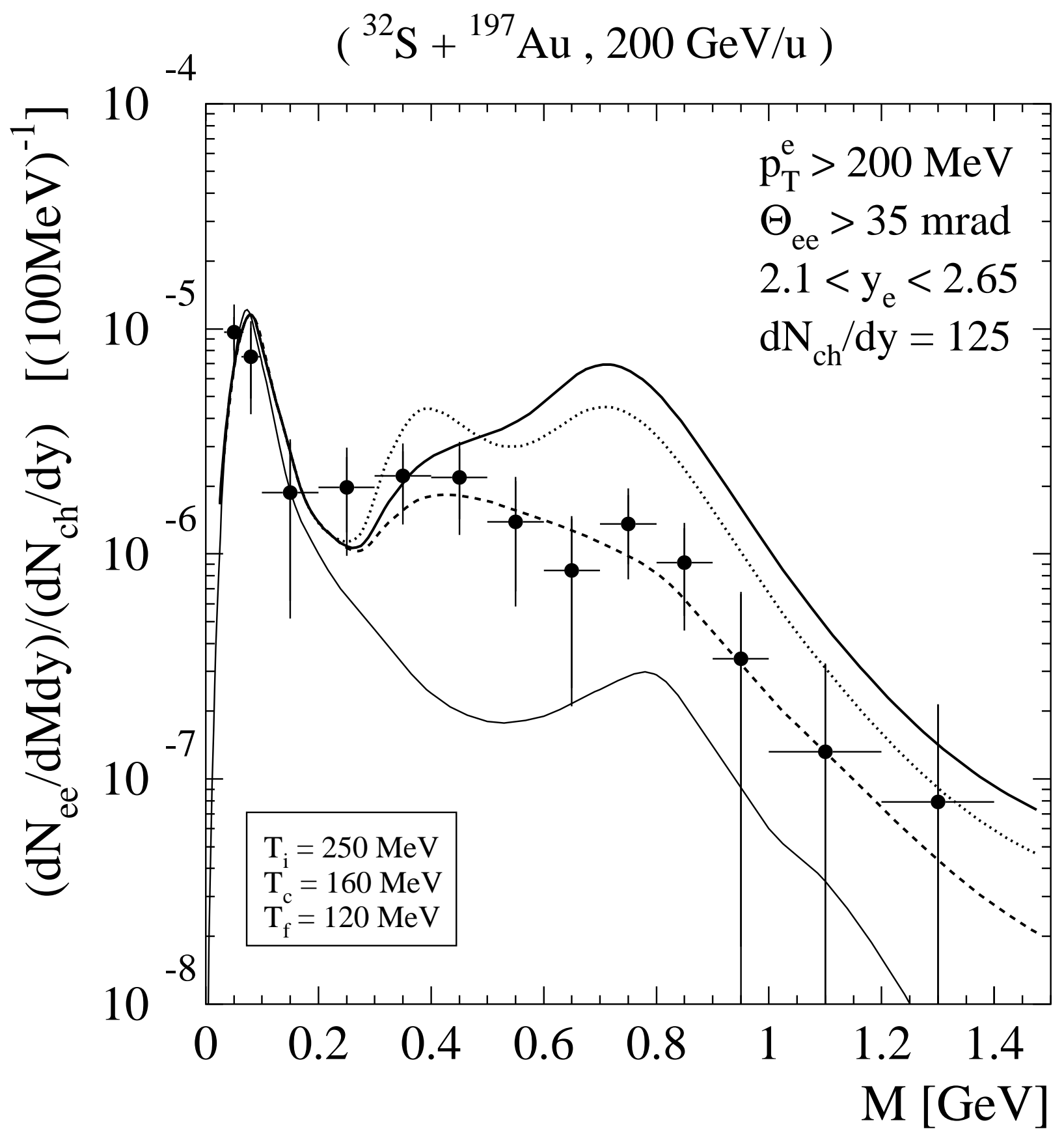

FIG. 3. The dilepton mass spectrum with an in-medium modification of the pion form factor and $T_{f}=120 \mathrm{MeV}$. The dashed curve shows the result with a temperature dependent width, and the dotted curve with a temperature dependent rho mass, as explained in the text. For comparison the full curve based on the vacuum pion form factor is also shown again. The hadron decay contribution (thin line) has been added to our pion annihilation results. 


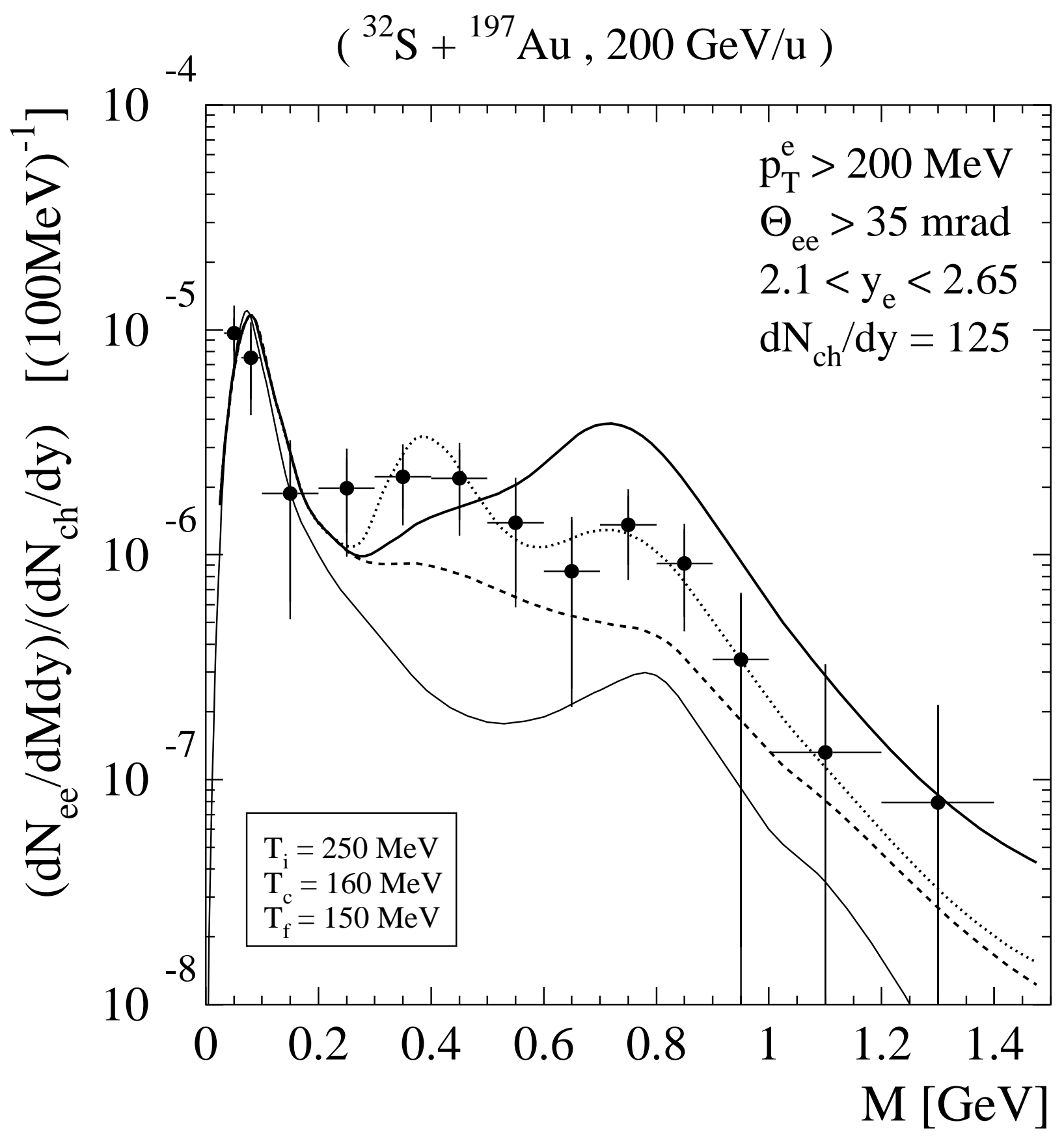

FIG. 4. Same as Fig. 3, but with a temperature $T_{f}=150 \mathrm{MeV}$. 